Ciencia y Educación, Vol. 4, No. 3, septiembre-diciembre, 2020

ISSN (impreso): 2613-8794•ISSN (en línea): 2613-8808

DOI: https://doi.org/10.22206/cyed.2020.v4i3.pp45-64

\title{
Aprendizaje situado con dispositivos móviles en contextos artísticos: estudio descriptivo con estudiantes universitarios
}

\author{
Situated learning with mobile devices in artistic contexts: \\ descriptive study with university students
}

\author{
Alfredo José Ramón-Verdúa ORCID: 0000-0002-8176-4146 \\ José Víctor Villalba-Gómez ${ }^{\mathrm{b}}$ ORCID: 0000-0002-7696-4549
}

Recibido: 27/02/2020 • Aprobado: 17/06/2020

Cómo citar: Ramón-Verdú, A. J., \& Villalba-Gómez, J. V. (2020). Aprendizaje situado con dispositivos móviles en contextos artísticos: estudio descriptivo con estudiantes universitarios. Ciencia y Educación, 4(3), 45-64. Doi: https://doi.org/10.22206/ cyed.2020.v4i3.pp45-64

\section{Resumen}

El presente artículo muestra los resultados de investigación de un proyecto educativo mediado por dispositivos móviles en dos exposiciones artísticas, con alumnado del Grado en Educación Primaria de la Universidad de Murcia. La muestra fue de 119 alumnos de tres especialidades. El objetivo fue evaluar una experiencia de innovación docente desde la percepción del alumnado, utilizando una metodología educativa basada en el diálogo mediado. Se realizó la evaluación de la experiencia mediante un test de rendimiento típico de tipo Likert, compuesto por cinco dimensiones: la interacción del alumno con la obra, con el espacio, las personas, los dispositivos móviles y su futura labor profesional. Los resultados evidencian que los valores obtenidos oscilan entre una valoración positiva y muy positiva. Se ha valorado muy satisfactoriamente el uso de dispositivos móviles en contextos artísticos dinámicos, dado que fomentan la participación, la reflexión y la manifestación de opiniones en tiempo real.

Palabras clave: educación artística; aprendizaje activo; aprendizaje móvil; contexto de aprendizaje; TIC.

a Universidad de Murcia, España. Correo-e: alfredoramon@um.es

b Universidad de Murcia, España. Correo-e:josevictor.villalba@um.es

\begin{abstract}
This article shows the research results of an educational project mediated by mobile devices, carried out in two artistic exhibition spaces with students from Primary Education Degree of the University of Murcia. The sample was of 119 students belonging to three different specialties. The objective was to evaluate a teaching innovation experience from the students' perception, using an educational methodology based on mediated dialogue. The evaluation of the experience was made through a typical performance test measured with a Likert-type scale, composed of five dimensions: the student's interaction with the work, with space, people, mobile devices and their future professional work. The results show that the obtained values oscillate between a positive and a very positive evaluation. The use of mobile devices in dynamic artistic contexts has been highly valued, as they encourage participation, reflection and the expression of opinions in real time.
\end{abstract}

Keywords: art education; activity learning; mobile learning; educational environment; ITC. 


\section{Introducción}

La utilización de los dispositivos móviles en educación está muy extendida hoy día. Uno de los principales retos para los docentes sigue siendo cómo utilizar estos medios tecnológicos para que trasciendan la barrera de lo instrumental — búsqueda de información, redes sociales, fotografía, video o el uso de aplicaciones_- para adentrarse en aspectos educativos de orden superior utilizando las herramientas disponibles en estos dispositivos. Entendido el aprendizaje móvil como un proceso, Sharples, Taylor y Vavoula (2007, p. 225) lo definieron como "los procesos para llegar a conocer a través de conversaciones en múltiples contextos entre personas y tecnologías interactivas personales". Estos contextos nos llevan a pensar en los espacios y en las situaciones en los que el aprendizaje es posible. Naismith, Lonsdale, Vavoula y Sharples (2004) definen el aprendizaje situado como el que se produce de forma particular en contextos sociales de participación, en situaciones reales y en contextos auténticos de experimentación participativa, aspectos que se van a tratar como eje central en este documento al abordar situaciones y tecnologías móviles como instrumentos para el aprendizaje sobre experiencias directas.

En la enseñanza de materias y disciplinas cercanas a las artes plásticas y visuales, el campo de la percepción supone uno de principales espacios sobre los que trabajar, ya que, al estar muy ligado a los contextos y a las experiencias de los autores y de los observadores, no existe una única respuesta a las cuestiones artísticas que se plantean. Esto condiciona en gran medida el diseño de las propuestas educativas dentro de contextos artísticos interpretables como refleja el estudio realizado por Kearney, Burden y Schuck (2019), en el que solo uno de los estudios que analizaron estaba relacionado con las artes visuales, aunque en ningún momento se habla de los procesos perceptivos.

Dentro de las estrategias de aprendizaje mediadas por dispositivos móviles en entornos artísticos y museísticos, destacan las dirigidas hacia la actividad dentro del paradigma de "aprender haciendo" inte- ractuando con los objetos y con el espacio. Con este planteamiento hay trabajos interesantes, como los de Kali, Sagy, Kuflik, Mogilevsky y Maayan-Fanar (2015); Cahill, Kuhn, Schmoll, Lo, McNally y Quintana (2011); o Aguayo, Eames y Cochrane (2020), en los que plantean frameworks para que los alumnos trabajen sobre contenidos en espacios museísticos; indagando, reflexionando y expresando después los resultados de sus actividades, dentro de un marco de elección múltiple. En ellos están presentes implícitamente los procesos cognitivos que los alumnos manejarán para realizar las tareas, aunque no inciden tanto sobre cuál ha sido la experiencia del alumno para obtener una visión holística del aprendizaje, indagando en los significados que cada uno de ellos ha extraído para sí.

En el campo de la enseñanza y del aprendizaje se suelen considerar dos procedencias distintas de los procesos educativos: uno de elaboración interna, que proviene del propio estudiante, constituido por sus experiencias, conocimientos previos y motivaciones particulares, y otro de origen externo, que proviene de los contextos en los cuales se desenvuelve y realizan las actividades. Zapata-Ros (2015), introduciendo los medios digitales para construir el conocimiento, define el aprendizaje como:

Un cambio o un incremento en las ideas (o material cognitivo, en los conocimientos y representaciones mentales) duradero y con repercusión en la práctica (operativa o potencial), y eventualmente en la conducta, que se produce como consecuencia de la experiencia del aprendiz, de su madurez o de la interacción con el entorno (social, de información y de medios) (p. 11).

Esto mismo ocurre en el aprendizaje de las artes plásticas y visuales, que si bien los procesos de aprendizaje son similares a las de otras disciplinas, se coloca el énfasis en las capacidades de los creadores para utilizar lenguajes, en muchos casos abstractos e interpretativos, y en el desarrollo de las capacidades para su lectura, comprensión y experimentación en contextos sociales concretos. Un planteamiento educativo con connotaciones artísticas de experimentación activa, se integra en el sistema expuesto 
en la Teoría de la Actividad planteada por Vygotsky (1978), y desarrollada posteriormente por Engenström (1987). Aquí se relaciona el aprendizaje de las personas cuando interaccionan entre sí en espacios comunicativos socializados, y cuando interaccionan con los contextos mientras realizan acciones mediadas por artefactos. En el campo artístico, esta triple interacción permite abordar el desarrollo de habilidades creativas y comunicativas dentro de una amplia gama de posibilidades tecnológicas, ya que "...el conocimiento en los entornos tecnológicos digitales, en los que se procesa la información y de la comunicación, (...) tiene lugar actividad social de los individuos conectados." (Zapata-Ros, 2015, p. 12). Esto ofrece un lugar a las tecnologías móviles y a las posibilidades de los espacios de aprendizaje no formales, y a los diálogos con finalidad educativa que allí se producen (Laurillard, 2007).

En contextos de enseñanza no formal, como pueden ser los artísticos, a menudo son los espacios en dónde se realizan experiencias difíciles de encontrar en las aulas tradicionales. Las metodologías suelen estar descentralizadas respecto a los espacios de aprendizaje habituales (Huerta, 2019), y sumando la tecnología, esta permite afrontar la enseñanza de las artes desde posiciones experimentales poco convencionales, al facilitar y profundizar en el desarrollo de actitudes analíticas y críticas dentro del campo de la percepción individual, dominada en ocasiones por los estados emocionales propios.

Es en esta confluencia de espacios es donde se desenvuelve el marco de referencia del estudio realizado: un espacio expositivo artístico, un espacio personal, y un espacio virtual tecnológico móvil, relacionado con Koole (2009) y su modelo para enmarcar el aprendizaje móvil. Se relacionan las personas que intervienen, permitiéndoles “...planificar y generar los cambios cualitativos (incluido el diseño y la implementación de las tecnologías) en sus contextos de vida" (Engenström, 1987, p. 24). Se establece de esta forma una relación entre el espacio, las personas y los medios; conformando una versión de aprendizaje situado y contextual, consolidándose como generador de experiencias intelectuales. Se centra en estos contextos no formales y en el interés de saber cómo se perciben los espacios y las obras después de realizar actividades de aprendizaje en los espacios generadores de experiencias. La motivación principal ha sido la de potenciar este tipo de actividad de aprendizaje, y obtener datos sobre la percepción y algunos de los procesos que han ocurrido trabajando en espacios inesperados y sugerentes, planteando la práctica educativa artística como un diálogo mediado por la tecnología móvil en un contexto generador de experiencias. Esto facilitaría a estudiantes poco interesados, pero altamente expectantes, una profundización en lo perceptivo y en lo intelectual al participar en una experiencia enriquecedora.

En este sentido, según diversas investigaciones se está produciendo un distanciamiento de las salas de exposiciones y de los museos, reflejo del consumo cultural mediante aparatos de comunicación masiva a la carta (Rosas, 2017). Esta afirmación está avalada por la última encuesta realizada en España sobre los hábitos y prácticas culturales entre 2018 y 2019 (Ministerio de Cultura y Deporte, 2019). Aquí se dice que el 70,2\% de los encuestados no han visitado una exposición en más de un año, y el $84 \%$ una galería de arte, aunque es verdad que han mejorado algo los porcentajes respecto a años anteriores, aumentando también el acceso a la cultura mediante dispositivos digitales. Estos datos se han visto reflejados en las conversaciones mantenidas de manera informal con los alumnos objeto de estudio; sorprendentemente, un importante número de ellos no asiste de manera habitual a espacios artísticos de este tipo, afirmando incluso alguno, que jamás había visitado una sala de exposiciones.

Patten, Arnedillo, y Tangney (2006) ya argumentaban cómo a través del aprendizaje móvil en contextos históricos y culturales, se puede afrontar la enseñanza sobre experiencias en las que la ubicación es determinante. Es en estos contextos donde emanan los motivos de aprendizaje artísticos (contextos escultóricos, pictóricos, sonoros, audiovisuales, fotográficos, performativos, espaciales, etc.), si se consideran 
artefactos experimentables dentro de la dimensión vygotskiana que se ha comentado, provocando, en el mejor de los casos, desarrollos en la percepción y en la metacognición de las personas participantes (Cahill, 2011). Estos artefactos encierran matices para observar y sentir, para darle un significado total a la actividad de aprendizaje al ser “...instrumentos que mediatizan las relaciones entre profesor y alumnos, o entre alumnos y saber" (Rickenmann, 2007, p. 2), para una co-construcción cognitiva e intelectual. Estos lugares de aprendizaje compartido configuran un constructivismo sociocultural a través de la observación, la imitación y la mediación con otros alumnos (Kali et al., 2015).

En actividades museísticas y expositivas en las que el tiempo es determinante para experimentar las obras, Rainoldi, Neuhofer y Jooss (2018) relacionan el factor tiempo de visionado con la atención visual, o atención sostenida. De este aspecto derivará el tiempo de atención, comprensión y análisis, características muy importantes en los procesos cognitivos de orden superior (Bernabéu, 2017). En 1952 Welford ya hablaba de que los tiempos, los diálogos, y la atención focalizada debían estar perfectamente organizados para evitar que el observador desestimase la información sin haberla comprendido ni experimentado. Teniendo esto presente, en la experiencia realizada se utilizaron los dispositivos móviles para gestionar los tiempos y las dinámicas participativas, considerando lo expuesto por Kearney et al. (2019) sobre cómo debe ser el aprendizaje móvil inteligente:

1. Altamente situado y auténtico.

2. Adaptativo y receptivo a los hábitos y comportamientos de aprendizajes cambiantes.

3. Centrado en el alumno, autodirigido y fortalecedor.

4. Altamente personalizado y adaptado al individuo.

5. Interactivo y dinámico.
6. Altamente contextual.

7. Colaborativo, interdependiente y altamente social.

Estos autores (Kearney et al., 2019) insisten en que el aprendizaje individualizado no excluye ni le resta importancia al aprendizaje socializado mientras se interacciona con los compañeros. En esta cuestión, el factor innovador sobre el que trabajamos relaciona el espacio individualizado, con el espacio social representado mediante la interacción física con las salas de exposiciones, compañeros y profesor-, y con los diálogos virtual, verbal y corporal como formas de expresión.

La comunicación instantánea móvil es la que permite la conversación en tiempo real con el profesor, presente en la sala, administrando los tiempos, los espacios y los conceptos artísticos just in time, utilizando la plataforma de preguntas y respuestas Socrative (2020), para modular su discurso de forma flexible. Esto permite adaptarlo según discurra la experiencia, oscilando entre una actividad individualizada y una actividad socializada, con una baja distancia transaccional (Park, 2011). "En educación, las innovaciones disruptivas crean nuevas prácticas, propósitos y procesos (...), nuevas relaciones entre estudiantes y maestros, y potencialmente un cambio en la naturaleza de la escuela y su relación con la comunidad" (Kearney et al., 2019, p. 6). El estudio de casos en un aprendizaje situado se convierte aquí en una estrategia muy valiosa (Naismith et al., 2004; Keskin y Metcalf, 2011; Poikela, Vuoskoski y Kärnä, 2009; Wang y Shen, 2012), para el estudio de las artes plásticas y visuales.

Hasta ahora se han expresado los factores que intervienen dentro de un contexto educativo centrado en el alumno, con dispositivos móviles como medio de trabajo y de comunicación. Factores de diversa índole, principalmente educativos, tecnológicos, espaciales y sociales, entre los que se generan relaciones en mayor o menor medida dependiendo de la estrategia educativa que se lleve a cabo. Estas relaciones se materializan mediante procesos interactivos de diálogo dinámico, 
y de retroalimentación fluida en tiempo real. A partir de estas dinámicas se generaron las actuaciones que se expresan a continuación.

\section{Metodología}

\section{Objetivos}

Para el presente trabajo se planteó el objetivo de analizar una experiencia de innovación docente, basada en técnicas de aprendizaje situado conversacional con dispositivos móviles en contextos artísticos, indagando sobre las opiniones de los estudiantes y su percepción durante la ejecución de la tarea. A partir de aquí se analizarían los datos obtenidos para determinar el grado de valor atribuido a dicha tarea, desde la propia experiencia del aprendiz.

\section{Enfoque y tipo de estudio}

Para lograr el objetivo propuesto se planteó un estudio exploratorio descriptivo de tipo cuantitativo, con un diseño de la estrategia metodológica de desarrollo transversal, dado que participaron tres grupos distintos en una unidad de tiempo concreto. Estos tres grupos conformaron un muestreo no probabilístico de tipo causal o incidental (Vieytes, 2004). La intervención se realizó en dos fases diferenciadas: una relativa a la propia actividad educativa y a la experiencia de aprendizaje que conlleva — la cual se explicará en el apartado Contexto de trabajo-, y otra relativa a la toma de datos sobre dicha experiencia educativa, y que será la que contenga el análisis de la investigación que se ha realizado.

\section{Muestra}

La muestra se seleccionó de entre los alumnos del Grado de Educación Primaria de la Universidad de Murcia. Se seleccionaron tres de las menciones del cuarto curso: mención de Lengua Extranjera Inglés, Lengua Extranjera Francés y Educación Intercultural, dentro de la asignatura Desarrollo del Lenguaje Visual y Plástico, impartida entre los meses de septiembre y diciembre de 2019. Los criterios para la inclusión del grupo de estudio están definidos por una muestra de estudiantes de corte no probabilístico incidental, ya que la muestra se seleccionó de forma directa e intencionalmente por conveniencia y accesibilidad a ella durante un intervalo temporal concreto, pudiéndose considerar representativa de la población sobre la que se tiene interés en la investigación (Sabariego y Bisquerra, 2009). La muestra representa el 20,51 $\%$ del total del alumnado del cuarto curso del grado, que en el curso 2019/2020 contaba con una población de 580 sujetos. El tamaño muestral quedó constituido por finalmente 119 individuos, divididos en tres grupos bastante equilibrados en número, siendo 98 de sexo femenino y 21 de sexo masculino.

\section{Técnicas e instrumentos}

La toma de datos se realizó mediante un cuestionario de rendimiento típico, creado ex profeso para el estudio, sobre una escala numérica continua de tipo Likert. Esta está compuesta por una gradación en 5 niveles, en la que 1 es el grado de mínima satisfacción y 5 el de máxima, según el grado estimativo de valor que le atribuya a cada una de las cuestiones. Está compuesto por un total de 16 preguntas centradas en la actividad, y distribuidas con base en cinco dimensiones que interesaba conocer: interacción con la obra (3 ítems), interacción con el espacio (3 ítems), interacción entre los presentes ( 4 ítems), interacción con el dispositivo móvil (5 ítems) y percepción de la actividad para su futuro profesional (1 ítem).

Se realizó la validación del cuestionario con base en el juicio de tres expertos en el área específica de desarrollo de la propuesta, y según la actividad que se pretendía evaluar, aportando opiniones de aspecto cualitativo en función de la suficiencia, claridad, coherencia y relevancia de las cuestiones planteadas. Se realizó la modificación de varias de ellas, pasando a realizar un piloto $(n=12)$, representando aproximadamente un $10 \%$ de la muestra inicial, y obteniendo un resultado coherente en cuanto a la uniformidad y comprensión de lo planteado en cada una de las 
dimensiones. Se determinó la fiabilidad utilizando el programa para análisis estadístico SPSS versión 24 para Windows, mediante un análisis de la consistencia interna del cuestionario (o la correlación interna de los ítems) utilizando el coeficiente de fiabilidad $\alpha$ de Cronbach, con un resultado de $\alpha=0.811$; por lo que se puede decir que el instrumento de recogida de información utilizado en la investigación es fiable.

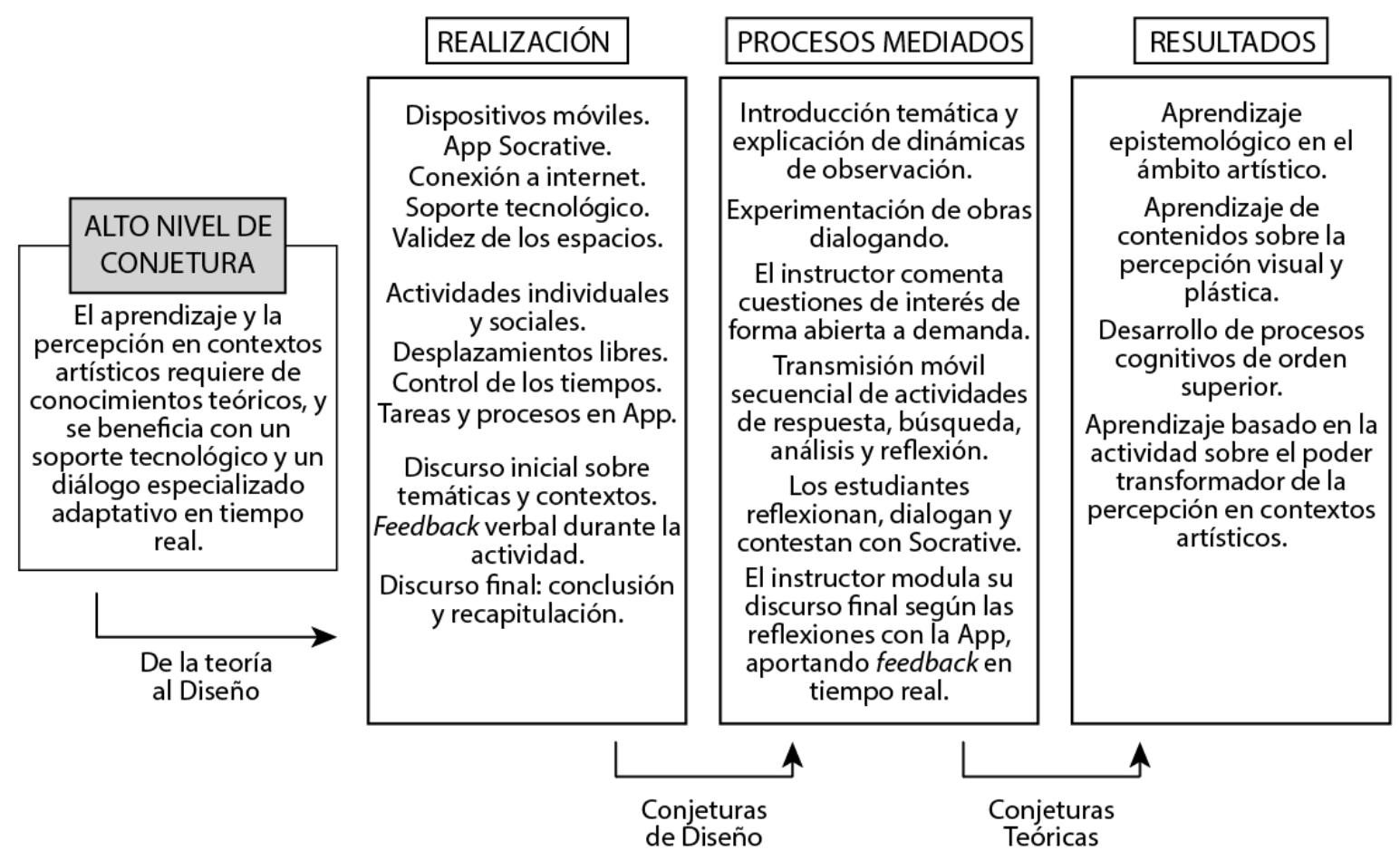

Figura 1. Mapa de conjeturas de la propuesta educativa en la investigación

\section{Contexto de trabajo}

El diseño de la actividad educativa se planteó como una secuencia de aprendizaje basada en el enfoque sobre conjeturas de Sandoval (2014), para organizar cómo puede hacerse realidad el aprendizaje partiendo de hipótesis que no se materializarán mientras no se realice la actividad. El planteamiento realizado se puede ver en la figura 1 : 


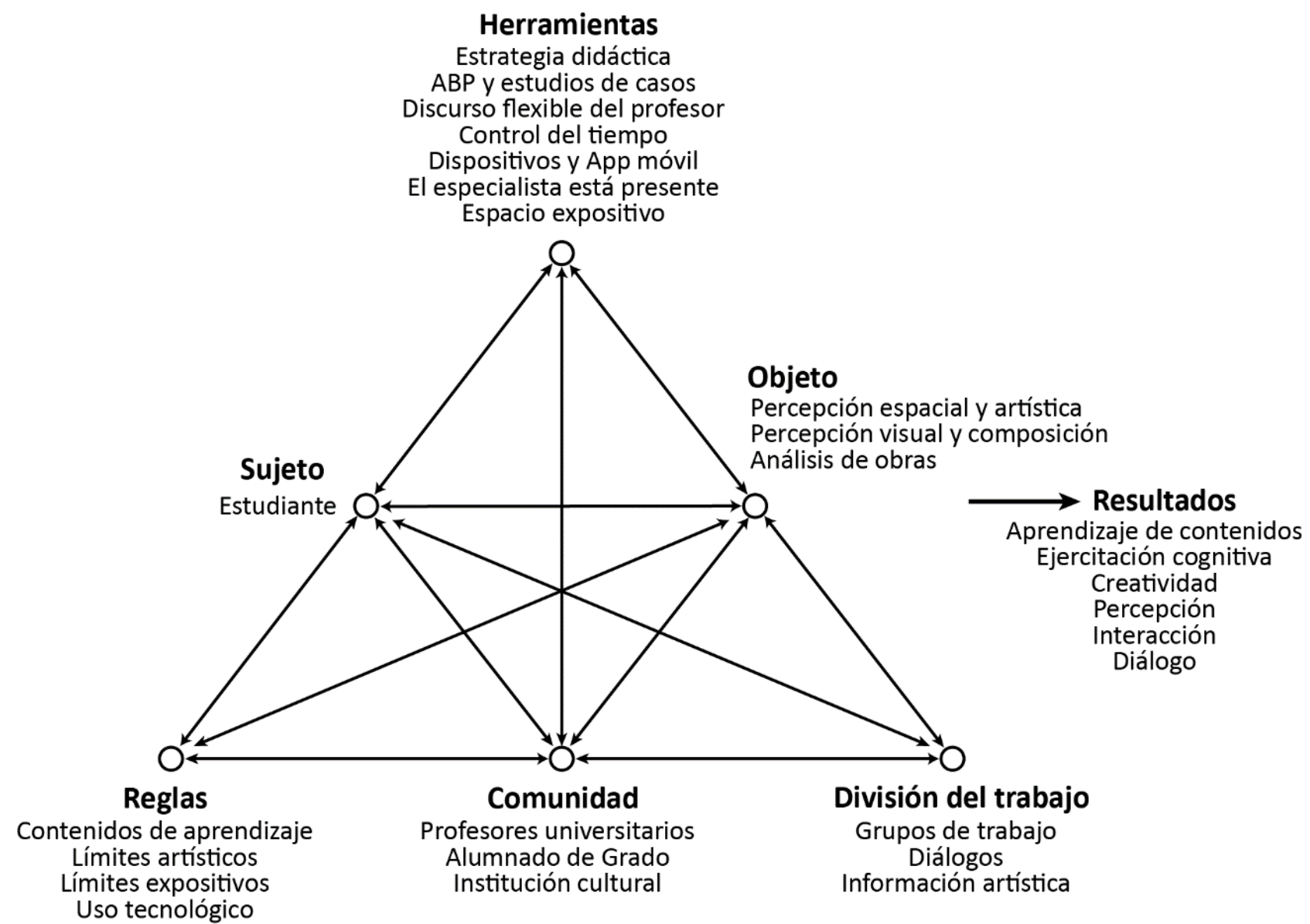

Figura 2. Diagrama del sistema de actividad de la práctica realizada por el estudiante

Fuente: basado en el modelo de Engenström (1987).

Para iniciar la actividad se convocó a los alumnos en la entrada y se dio una información sobre los espacios y las obras. A continuación, se explicó que la dinámica estaba basada en una actividad de reconocimiento, búsqueda, y reflexión, que dejarían registrada a través de los dispositivos móviles. Durante la ejecución de la actividad, el profesor estaría realizando una observación participante, respondiendo de forma dinámica a las circunstancias y a las cuestiones que en todo momento se fueron dando, facilitando la comprensión de los conceptos tratados. En esta observación, se fueron activando digitalmente las preguntas que dirigirían a los estudiantes hacia las observaciones y reflexiones que interesaba que hiciesen, en momentos concretos previamente marcados, observando dinámicamente y en tiempo real las respuestas que por escrito iban dando los alumnos.

Los espacios en donde se ha realizado la investigación han sido: Sala Verónicas (Espacio 1) y Palacio Almudí (Espacio 2), ambos en la ciudad de Murcia.

El Espacio 1 es una iglesia barroca del siglo XVIII con planta de cruz latina, originariamente anexo a un convento de clausura que actualmente no existe, conservando los elementos arquitectónicos de su función original, adaptado a propuestas de arte contemporáneo. En este sentido, permanecen intactos elementos tales como rejas, ventanas (tapiadas) y 
balcones. El espacio está exento de luz natural, por lo que precisa de iluminación artificial que es minuciosamente adaptada a cada muestra. El espacio es muy versátil, en el que tanto suelo, techo y paredes ofrecen posibilidades expositivas interesantes, traducidas normalmente en instalaciones artísticas. La exposición, denominada Correspondencias, comisariada por Jesús Alcaide, muestra esculturas de los artistas Pepe Espaliú y Juan Muñoz, habiéndose preparado a propósito para que las obras dialoguen con la naturaleza del espacio. Los dos artistas son grandes referencias espańolas de la escultura del siglo xx. Según Alcaide (2019), se trata de un diálogo con ecos de la propia historia del lugar, donde conviven balcones y jaulas, adoquines y pasamanos, tortugas y elementos en equilibrio, donde se produce un armonioso juego de luces y sombras. Finalmente, Alcaide denomina la exposición como un lugar en el que se encuentran dos artistas y conviven elementos comunes y diferentes maneras de trabajar.

El Espacio 2 es un edificio del siglo xv adaptado actualmente como sala de exposiciones, después de haber tenido diversos usos. Aunque se trata de un espacio que podría ser versátil para cualquier tipo de obra, está acondicionado fundamentalmente para obras de índole tradicional: pintura y escultura. En este caso, la muestra se denomina "París Vivant". Es un conjunto de obras pictóricas pertenecientes a pintores de principios del Siglo xx, la mayoría de ellos ligados a la Escuela de París, artistas como Gustave Madelain, Marcel Cosson, Leon Suzanne, Augustin Grass-Mick, Joaquim Sunyer, Alphonse Quizet, Henri Ottmann, Pierre Farge, Amedeo Modigliani y Pedro Flores, entre otros. La exposición está comisariada por Juan Pérez Ferra, y según la entrevista de Carrión (2019):

Cada una de las 67 obras que se seleccionaron para la ocasión, tiene detrás una historia que plasma la vida de París y las relaciones entre los centenares de artistas de la época, muchos de los cuales quedaron eclipsados por grandes maestros como Picasso, Braque, Matisse o el propio Modigliani.
Con relación a las dinámicas didácticas, en el Espacio 1 se plantea el objetivo de llevar a la práctica herramientas cognitivas de orden básico, incentivando los procesos de percepción, sensación, atención, y memoria. Respecto al Espacio 2, el objetivo marcado es el de potenciar el desarrollo de procesos cognitivos de orden superior, incentivando al alumnado a ejecutar acciones de pensamiento, lenguaje, y lenguaje visual, además de diversas funciones ejecutivas.

En ambos espacios se estableció un tiempo aproximado de veinte minutos para que el alumno realizase cada uno de los recorridos, sin premisas condicionantes, iniciándose en pequeños grupos un proceso activo de interacción con la obra y el lugar. Este momento lo podemos denominar "tiempo de contextualización o calentamiento", donde el alumno ya comienza a percibir distintos elementos del lenguaje visual. Sin embargo, como es habitual en nuestro alumnado, la duración de estos momentos suele ser extremadamente corto. Es a partir de aquí cuando se inicia el núcleo central del método, provocando una interacción y diálogo entre los diversos agentes presentes: el espacio, la obra, el alumnado, la tecnología y el docente.

La herramienta utilizada para la interacción es la app móvil Socrative (2020), utilizada en cada uno de los espacios en sus dos versiones, la versión Teacher para el docente, y la versión Student para el alumnado. Esta aplicación, gratuita, permite que el docente comparta recursos, imágenes, tareas, y cuestiones; con las que el estudiante pueda responder de forma inmediata. El profesor interviene como moderador, controlando los tiempos, y utilizando en tiempo real la información que el alumnado le proporciona. De esta forma el docente puede interactuar en cualquier momento, adaptando su discurso a la reacción y capacidad del alumno.

Los objetivos didácticos se plantearon mediante tareas y cuestiones que se activaron secuencialmente en cada uno de los espacios, mediante la aplicación móvil comentada. Estas se mostraron en forma de cuestionarios individuales de rendimiento óptimo con respuestas abiertas, cortas y contextualizadas, 
como se puede ver en las tablas 1 y 2 , aunque alguna de ellas (cuestión 3) se debía completar acabada la actividad. El objetivo de estos cuestionarios es el de guiar la actividad de aprendizaje, sugiriendo e incentivando al alumnado a observar y moverse detenidamente el espacio. Las cuestiones están relacionadas con contenidos de la asignatura Desarrollo del Lenguaje Visual y Plástico, tales como la percepción, el lenguaje visual y los espacios del arte contemporáneo como entorno educativo.

Tabla 1. Cuestiones educativas a resolver por el alumnado en el Espacio 1

1. Si nos centramos en las jaulas. ¿Qué significado tienen para ti? ¿Qué podrías decir sobre las sombras? ¿Crees que el espacio en el que se muestran afecta a su interpretación? ¿Por qué?

2. ¿Crees que en la exposición hay alguna obra que no encaja?

3. ¿Qué significado general encierra la exposición?

4. ¿Estableces alguna relación entre la exposición y la vida real?

5. ¿Te ha supuesto alguna dificultad reflexionar sobre el contenido de la exposición?

Fuente: elaboración propia.
Tabla 2. Cuestiones educativas a resolver por el alumnado en el Espacio 2

1. Fotografía y justifica después en tu dosier, obras que, según tú, representen lo siguiente:
a. Equilibrio
b. Punto de atención
c. Convergencia de direcciones visuales
d. Ley de Pregnancia o de la buena forma
e. Principio de la marcha

2. Busca al pintor murciano Pedro Flores. Desde el conocimiento que tienes del lenguaje visual y plástico, dame tu opinión sobre sus obras.

3. Para realizar en grupo de trabajo. Hay que seleccionar una de las obras y hacer un breve análisis desde tres perspectivas:

a. Factores formales: relaciones geométricas, tensiones dinámicas, composición, pesos visuales, centros de la obra, equilibrio, formas geométricas reconocibles, color, técnica.

b. Factores asociativos: relaciones conceptuales, recuerdos, conocimientos y asociaciones generales a los que la obra nos induce.

c. Factores de contenido: interpretación simbólica, significados, interpretación denotada y connotada. (El contenido denotado se refiere a los elementos explícitos ofrecidos por la imagen. El contenido connotado se refiere a los mensajes no explícitos que aparecen en una lectura compleja de la imagen).

Fuente: elaboración propia.

Una vez realizados los ejercicios de la actividad didáctica de las tablas 1 y 2 , se abordó la fase relativa a la toma de datos de investigación, mediante un cuestionario que el alumnado completó.

\section{Procedimientos de recolección}

El cuestionario fue creado digitalmente en el repositorio de Google Drive, a partir del cual se generó un enlace que fue enviado al alumnado a través del Aula Virtual de la Universidad de Murcia. El cuestionario 
fue cumplimentado mediante dispositivos móviles durante los dos primeros días de la primera semana de noviembre de 2019, tras la realización de la experiencia de innovación docente, recogiendo los datos de los 119 encuestados.

Los datos introducidos se exportaron a una matriz en Microsoft Excel, para ser importados posteriormente a SPSS Statistics versión 24 de IBM, donde se realizó el tratamiento de datos, generando los reportes estadísticos descriptivos, tablas de frecuencia y de contingencia.

\section{Resultados}

En la tabla 3 se pueden ver los datos estadísticos relativos a las dimensiones establecidas. Los mínimos y los máximos varían según el número de ítems. Se observan desviaciones estándar bajas hacia moderadas, lo que implica cierta dispersión respecto al promedio de las puntuaciones. Las medias están cercanas al máximo, lo que induce a pensar, inicialmente, que las puntuaciones han tendido hacia valores altos.

Tabla 3. Datos estadísticos descriptivos de las dimensiones estudiadas

\begin{tabular}{|l|c|c|c|c|c|}
\hline \multicolumn{1}{|c|}{ Dimensión } & $N$ & Minimo & Máximo & Media & Desviación estándar \\
\hline $\begin{array}{l}\text { Interacción del } \\
\text { alumno con la obra }\end{array}$ & 119 & 7,00 & 15,00 & 12,3782 & 1,81795 \\
\hline $\begin{array}{l}\text { Interacción del } \\
\text { alumno con el espacio }\end{array}$ & 119 & 7,00 & 15,00 & 12,6218 & 1,87305 \\
\hline $\begin{array}{l}\text { Interacción con las } \\
\text { personas }\end{array}$ & 119 & 12,00 & 20,00 & 17,1681 & 1,79131 \\
\hline $\begin{array}{l}\text { Interacción con el } \\
\text { dispositivo móvil }\end{array}$ & 119 & 14,00 & 25,00 & 21,6387 & 2,69881 \\
\hline $\begin{array}{l}\text { Aporte para futura } \\
\text { labor profesional }\end{array}$ & 119 & 2,00 & 5,00 & 4,2941 &, 66833 \\
\hline \begin{tabular}{l} 
N válido (por lista) \\
\hline
\end{tabular} & 119 & & & & \\
\hline
\end{tabular}

Fuente: elaboración propia.

A continuación, se muestran los datos estadís- los ítems que componen dichas dimensiones. ticos y de frecuencias de las diversas dimensiones y de 
Dimensión 1: Interacción del alumno con la obra

Se puede afirmar que, en gran medida, la actividad creada a través de Socrative incentiva a que el alumnado observe y experimente con detenimiento cada una de las obras, permitiéndole realizar un ejercicio de reflexión en el que profundiza en los aspectos conceptuales.

Tabla 4. Datos estadísticos descriptivos de la dimensión: Interacción del alumnado con la obra

\begin{tabular}{|l|c|c|c|c|c|c|c|}
\hline \multicolumn{1}{|c|}{ Ítems } & \multicolumn{2}{|c|}{$N$} & Media & Moda & $\begin{array}{c}\text { Desviación } \\
\text { estándar }\end{array}$ & Minimo & Máximo \\
\cline { 2 - 7 } & Válido & Perdidos & & & & & \\
\hline $\begin{array}{l}\text { La actividad incentiva que } \\
\text { observe y experimente con } \\
\text { detenimiento cada una de las } \\
\text { obras. }\end{array}$ & 119 & 0 & 4,10 & 4 &, 694 & 2 & 5 \\
\hline $\begin{array}{l}\text { La dinámica creada durante la } \\
\text { visita, me ha permitido profun- } \\
\text { dizar en los aspectos concep- } \\
\text { tuales de las obras. }\end{array}$ & 119 & 0 & 4,02 & 4 &, 813 & 2 & 5 \\
\hline $\begin{array}{l}\text { La actividad tiene sentido } \\
\text { lúdico y me genera atracción. }\end{array}$ & 119 & 0 & 4,26 & 5 &, 797 & 2 & 5 \\
\hline
\end{tabular}

Fuente: elaboración propia.

En la tabla 4 se evidencia la poca dispersión de los datos dentro de la dimensión, representado por la desviación estándar, y se constata la tendencia de las respuestas hacia valores medios y altos, tal y como se puede apreciar por el valor de la moda en cada uno de los ítems. A continuación, en las tablas 5, 6 y 7, se muestran las tablas de frecuencia de cada uno de los ítems que componen la dimensión.

Tabla 5. Tabla de frecuencia ítem 1

La actividad incentiva que observe y experimente con detenimiento cada una de las obras.

\begin{tabular}{|l|c|c|}
\hline \multicolumn{1}{|c|}{ Opción } & Frecuencia & Porcentaje \\
\hline Poco & 2 & 1,7 \\
\hline En cierta medida & 17 & 14,3 \\
\hline Bastante & 67 & 56,3 \\
\hline Mucho & 33 & 27,7 \\
\hline Total & 119 & 100,0 \\
\hline
\end{tabular}

Fuente: elaboración propia.
Tabla 6. Tabla de frecuencia ítem 2

\begin{tabular}{|c|c|c|}
\hline Opción & Frecuencia & Porcentaje \\
\hline Poco & 4 & 3,4 \\
\hline En cierta medida & 26 & 21,8 \\
\hline Bastante & 53 & 44,5 \\
\hline Mucho & 36 & 30,3 \\
\hline Total & 119 & 100,0 \\
\hline
\end{tabular}

Fuente: elaboración propia.

Tabla 7. Tabla de frecuencia ítem 3

\begin{tabular}{|l|c|c|}
\hline \multicolumn{2}{|c|}{ La actividad tiene sentido lúdico y me genera atracción. } \\
\hline \multicolumn{1}{|c|}{ Opción } & Frecuencia & Porcentaje \\
\hline Poco & 4 & 3,4 \\
\hline En cierta medida & 14 & 11,8 \\
\hline Bastante & 48 & 40,3 \\
\hline Mucho & 53 & 44,5 \\
\hline Total & 119 & 100,0 \\
\hline
\end{tabular}

Fuente: elaboración propia. 
En las tablas 5 y 6 destaca la frecuencia de la opción Bastante, siendo una de 67 y otra de 53, suponiendo prácticamente la mitad de las respuestas en cada una de ellas. Por el contrario, en la tabla 7 se puede apreciar que la opción Mucho es la que predomina, aunque la opción bastante, está bastante cerca.

\section{Dimensión 2: Interacción del alumno con el espacio}

Respecto a la interacción del alumnado con el espacio expositivo, más de dos terceras partes del alumnado verifica que la actividad con dispositivos móviles le ha permitido conocer mejor el lugar y cada uno de sus rincones. El contexto les ha ayudado a experimentar y a darle significado a la obra, comprendiendo de forma más cercana las distintas manifesta- ciones artísticas, en este caso, la nueva escultura del siglo xx y manifestaciones pictóricas precedentes, de principios de siglo. La metodología docente utilizada, en el propio lugar, ha ayudado al alumnado a entender el feedback de la intervención del docente con las obras expuestas.

En la tabla 8 se evidencia nuevamente poca dispersión de los datos, representado por la desviación estándar, y se vuelve a ver una tendencia de las respuestas hacia valores medios y altos. Los valores de la media y la moda, acompañados por una desviación estándar relativamente baja, reflejan datos cercanos, aunque el tercer ítem de la tabla señala un mínimo de 1 y comprobamos en la tabla 11 que se han respondido valores de toda la escala.

Tabla 8. Datos estadísticos descriptivos de la dimensión: Interacción del alumnado con el espacio

\begin{tabular}{|c|c|c|c|c|c|c|c|}
\hline \multirow[b]{2}{*}{ Ítems } & \multicolumn{2}{|c|}{$N$} & \multirow[b]{2}{*}{ Media } & \multirow[b]{2}{*}{ Moda } & \multirow{2}{*}{$\begin{array}{l}\text { Desvi- } \\
\text { ación } \\
\text { estándar }\end{array}$} & \multirow[b]{2}{*}{ Minimo } & \multirow[b]{2}{*}{ Máximo } \\
\hline & Válido & Perdidos & & & & & \\
\hline $\begin{array}{l}\text { El aprendizaje situado, es decir, en el lugar, me ha } \\
\text { ayudado a comprender el feedback del docente, integrando } \\
\text { así ideas acerca del arte actual. }\end{array}$ & 119 & 0 & 4,28 & 5 & ,769 & 2 & 5 \\
\hline $\begin{array}{l}\text { La actividad real, junto a la interacción con el entorno, es } \\
\text { determinante para la comprensión de la obra. }\end{array}$ & 119 & 0 & 4,24 & 4 & ,663 & 3 & 5 \\
\hline $\begin{array}{l}\text { El contexto me ha ayudado a experimentar y darle signifi- } \\
\text { cado a la obra. }\end{array}$ & 119 & 0 & 4,10 & 4 & ,887 & 1 & 5 \\
\hline
\end{tabular}

Fuente: elaboración propia.

A continuación, en las tablas 9, 10 y 11, se muesque componen la dimensión. tran las tablas de frecuencia de cada uno de los ítems

Tabla 9. Tabla de frecuencia ítem 4

\begin{tabular}{|l|c|c|}
\hline \multicolumn{1}{|l|}{ El aprendizaje situado, es decir, en el lugar, me ha ayudado a comprender el feedback del docente, integrando así ideas acerca del arte actual. } \\
\hline \multicolumn{1}{|c|}{ Opción } & Frecuencia & Porcentaje \\
\hline Poco & 3 & 2,5 \\
\hline En cierta medida & 14 & 11,8 \\
\hline Bastante & 49 & 41,2 \\
\hline Mucho & 53 & 44,5 \\
\hline Total & 119 & 100,0 \\
\hline
\end{tabular}

Fuente: elaboración propia. 
Tabla 10. Tabla de frecuencia ítem 5

\begin{tabular}{|l|c|c|}
\hline \multicolumn{3}{|l|}{$\begin{array}{l}\text { La actividad real, junto a la interacción con el entorno, es } \\
\text { determinante para la comprensión de la obra. }\end{array}$} \\
\hline \multicolumn{1}{|c|}{ Opción } & Frecuencia & Porcentaje \\
\hline En cierta medida & 15 & 12,6 \\
\hline Bastante & 60 & 50,4 \\
\hline Mucho & 44 & 37,0 \\
\hline Total & 119 & 100,0 \\
\hline
\end{tabular}

Fuente: elaboración propia.

Tabla 11. Tabla de frecuencia ítem 6

\begin{tabular}{|c|c|c|}
\hline Opción & Frecuencia & Porcentaje \\
\hline Nada & 1 & ,8 \\
\hline Poco & 5 & 4,2 \\
\hline En cierta medida & 20 & 16,8 \\
\hline Bastante & 48 & 40,3 \\
\hline Mucho & 45 & 37,8 \\
\hline Total & 119 & 100,0 \\
\hline
\end{tabular}

Fuente: elaboración propia.

En la tabla 11 se podría destacar que por primera vez existe una frecuencia que indique $N a d a$, aunque solo una única vez.

\section{Dimensión 3: Interacción con las personas}

Respecto a la interactuación del propio alumnado entre sí, y con el profesor, observando la tabla 12 , se puede decir que la metodología utilizada favorece la comunicación mientras analizan las exposiciones, dado que, aunque la desviación se mantiene sin llegar a 1, la moda sigue estando en valores altos (4 y 5), por lo que se evidencia que gran parte de las respuestas ponderan suficientemente bien la metodología empleada, valorando la comunicación en el lugar en tiempo real. Además, el alumnado revela, de forma general, que la actividad socializada favorece la comunicación con el docente.
Tabla 12. Datos estadísticos descriptivos de la dimensión: Interacción con las personas

\begin{tabular}{|l|c|c|c|c|c|c|c|}
\hline \multicolumn{1}{|c|}{ Ítems } & \multicolumn{2}{|c|}{$N$} & Media & Moda & $\begin{array}{c}\text { Desviación } \\
\text { estándar }\end{array}$ & Mínimo & Máximo \\
\cline { 2 - 6 } & Válido & Perdidos & & & & & \\
\hline $\begin{array}{l}\text { Es adecuado } \\
\text { que la actividad } \\
\text { aporte } \\
\text { información en } \\
\text { tiempo real al } \\
\text { docente, para que } \\
\text { pueda trabajar de } \\
\text { forma conjunta } \\
\text { y pública con } \\
\text { nosotros. }\end{array}$ & 119 & 0 & 4,43 & 5 &, 658 & 3 & 5 \\
\hline $\begin{array}{l}\text { La actividad } \\
\text { permite } \\
\text { interactuar con } \\
\text { los compańeros } \\
\text { mientras analizo } \\
\text { la exposición. }\end{array}$ & 119 & 0 & 4,50 & 5 &, 675 & 2 & 5 \\
\hline $\begin{array}{l}\text { La actividad } \\
\text { favorece la } \\
\text { comunicación } \\
\text { con el docente. }\end{array}$ & 119 & 0 & 4,27 & 4 &, 709 & 3 & 5 \\
\hline $\begin{array}{l}\text { El número de } \\
\text { alumnos que } \\
\text { han participado } \\
\text { es idóneo para } \\
\text { realizar esta } \\
\text { actividad. }\end{array}$ & 119 & 0 & 3,97 & 4 &, 848 & 1 & 5 \\
\hline
\end{tabular}

Fuente: elaboración propia.

En la tabla 16 se aprecia cierta disconformidad con el número de alumnos con el que se tuvo que hacer la actividad. En este caso, no se les preguntó por el número que considerasen el idóneo, pero es de suponer que, al haber sido grupos numerosos, la disconformidad se refiere a exceso de personas para poder interactuar con libertad. Las tablas de frecuencia de cada uno de los ítems son las que vienen a continuación.

Tabla 13. Tabla de frecuencia ítem 7

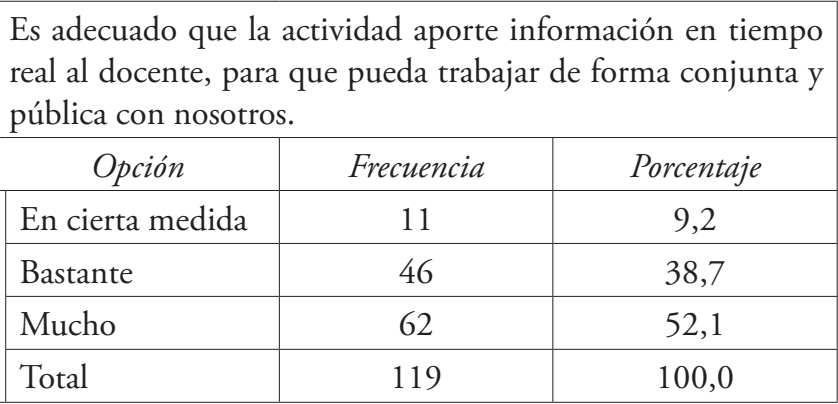

Fuente: elaboración propia. 
Tabla 14. Tabla de frecuencia ítem 8

\begin{tabular}{|l|c|c|}
\hline $\begin{array}{l}\text { La actividad permite interactuar con los compañeros mientras } \\
\text { analizo la exposición. }\end{array}$ \\
\hline \multicolumn{1}{|c|}{ Opción } & Frecuencia & Porcentaje \\
\hline Poco & 2 & 1,7 \\
\hline En cierta medida & 6 & 5,0 \\
\hline Bastante & 42 & 35,3 \\
\hline Mucho & 69 & 58,0 \\
\hline Total & 119 & 100,0 \\
\hline
\end{tabular}

Fuente: elaboración propia.

Tabla 15. Tabla de frecuencia ítem 9

\begin{tabular}{|l|c|c|}
\hline \multicolumn{1}{|c|}{ La actividad favorece la comunicación con el docente. } \\
\hline \multicolumn{1}{|c|}{ Opción } & Frecuencia & Porcentaje \\
\hline En cierta medida & 18 & 15,1 \\
\hline Bastante & 51 & 42,9 \\
\hline Mucho & 50 & 42,0 \\
\hline Total & 119 & 100,0 \\
\hline
\end{tabular}

Fuente: elaboración propia.

Tabla 16. Tabla de frecuencia ítem 10

\begin{tabular}{|l|c|c|}
\hline \multicolumn{3}{|l|}{$\begin{array}{l}\text { El número de alumnos que han participado es idóneo para } \\
\text { realizar esta actividad. }\end{array}$} \\
\hline \multicolumn{1}{|c|}{ Opción } & Frecuencia & Porcentaje \\
\hline Nada & 1 &, 8 \\
\hline Poco & 3 & 2,5 \\
\hline En cierta medida & 29 & 24,4 \\
\hline Bastante & 51 & 42,9 \\
\hline Mucho & 35 & 29,4 \\
\hline Total & 119 & 100,0 \\
\hline
\end{tabular}

Fuente: elaboración propia.

Las tablas 13, 14, 15 y 16 siguen reflejando conformidad sobre las cuestiones que se les pregunta, a pesar de que los ítems, en alguna medida, representando el término medio, han cobrado cierto protagonismo, aunque sin acercarse a los valores más positivos.

\section{Dimensión 4: Interacción con el dispositivo móvil}

Analizando la tabla 17, se puede interpretar que gran parte de los alumnos valora la oportunidad que les da la aplicación móvil de exponer su opinión de forma instantánea, y que sea de utilidad para el docente para utilizarlas inmediatamente en sus intervenciones. Las medias son bastante altas, todos los ítems poseen un máximo de puntuación, y la dispersión que ofrece la desviación estándar no es alta tampoco. La moda también indica que un gran número de respuestas están valoradas con 5 , la anotación más alta.

Tabla 17. Datos estadísticos descriptivos de la dimensión: Interacción con el dispositivo móvil

\begin{tabular}{|c|c|c|c|c|c|c|c|}
\hline \multirow[b]{2}{*}{ Ítems } & \multicolumn{2}{|c|}{$N$} & \multirow[b]{2}{*}{$\frac{\mathbb{Z}}{\mathbb{Z}}$} & \multirow[b]{2}{*}{$\frac{8}{8}$} & \multirow{2}{*}{ 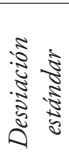 } & \multirow[b]{2}{*}{ 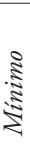 } & \multirow[b]{2}{*}{ 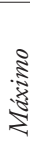 } \\
\hline & 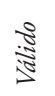 & $\frac{\sqrt{5}}{\frac{\pi}{\tilde{a}}}$ & & & & & \\
\hline $\begin{array}{l}\text { Reflexionar a través del } \\
\text { dispositivo móvil me ha } \\
\text { resultado fácil y adecuado. }\end{array}$ & 119 & 0 & 4,26 & 5 & ,786 & 2 & 5 \\
\hline $\begin{array}{l}\text { Valoro poder exponer mi } \\
\text { opinión de forma instantánea } \\
\text { mediante el móvil, y que } \\
\text { pueda tenerse en cuenta para } \\
\text { la explicación. }\end{array}$ & 119 & 0 & 4,36 & 5 & ,722 & 2 & 5 \\
\hline $\begin{array}{l}\text { La actividad con dispositivos } \\
\text { móviles me permite conocer } \\
\text { mejor el espacio expositivo, } \\
\text { incentivándome a explorar } \\
\text { sus rincones. }\end{array}$ & 119 & 0 & 4,01 & 4 & 907 & 2 & 5 \\
\hline $\begin{array}{l}\text { En el funcionamiento de la } \\
\text { actividad, la utilización del } \\
\text { móvil ha sido positiva. }\end{array}$ & 119 & 0 & 4,32 & 5 & ,724 & 3 & 5 \\
\hline $\begin{array}{l}\text { La app móvil me ha resul- } \\
\text { tado sencilla para llevar a } \\
\text { cabo la actividad. }\end{array}$ & 119 & 0 & 4,69 & 5 & ,579 & 3 & 5 \\
\hline
\end{tabular}

Fuente: elaboración propia. 
En este sentido, la actividad realizada con dispositivos móviles, al parecer, ha sido muy positiva. Como resultado general se puede inferir que la valoración del alumnado respecto a la utilización del teléfono móvil como herramienta en contextos artísticos, ha sido positiva, y la aplicación Socrative en concreto, les ha resultado sencilla y lúdica, generando gran atracción para realizar la actividad.

Las tablas de frecuencia de cada uno de los ítems se muestran a continuación, en las tablas 18, 19, 20, 21 y 22. Aunque todas indican una alta puntuación para todos los ítems preguntados, cabría destacar la tabla 22, dado que expresa valores muy altos para la cuestión relacionada con el uso de Socrative.

Tabla 18. Tabla de frecuencia ítem 11

Reflexionar a través del dispositivo móvil me ha resultado fácil y adecuado.

\begin{tabular}{|l|c|c|}
\hline \multicolumn{1}{|c|}{ Opción } & Frecuencia & Porcentaje \\
\hline Poco & 4 & 3,4 \\
\hline En cierta medida & 13 & 10,9 \\
\hline Bastante & 50 & 42,0 \\
\hline Mucho & 52 & 43,7 \\
\hline Total & 119 & 100,0 \\
\hline
\end{tabular}

Fuente: elaboración propia.

Tabla 19. Tabla de frecuencia ítem 12

Valoro poder exponer mi opinión de forma instantánea mediante el móvil, y que pueda tenerse en cuenta para la explicación.

\begin{tabular}{|l|c|c|}
\hline \multicolumn{1}{|c|}{ Opción } & Frecuencia & Porcentaje \\
\hline Poco & 2 & 1,7 \\
\hline En cierta medida & 11 & 9,2 \\
\hline Bastante & 48 & 40,3 \\
\hline Mucho & 58 & 48,7 \\
\hline Total & 119 & 100,0 \\
\hline
\end{tabular}

Fuente: elaboración propia.
Tabla 20. Tabla de frecuencia ítem 13

\begin{tabular}{|c|c|c|}
\hline \multicolumn{3}{|c|}{$\begin{array}{l}\text { La actividad con dispositivos móviles me permite conoce } \\
\text { mejor el espacio expositivo, incentivándome a explorar } \\
\text { rincones. }\end{array}$} \\
\hline Opción & Frecuencia & Porcentaje \\
\hline Poco & 7 & 5,9 \\
\hline En cierta medida & 27 & 22,7 \\
\hline Bastante & 43 & 36,1 \\
\hline Mucho & 42 & 35,3 \\
\hline Total & 119 & 100,0 \\
\hline
\end{tabular}

Fuente: elaboración propia.

Tabla 21. Tabla de frecuencia ítem 14

\begin{tabular}{|c|c|c|}
\hline \multicolumn{3}{|c|}{$\begin{array}{l}\text { En el funcionamiento de la actividad, la utilización del móvi } \\
\text { ha sido positiva. }\end{array}$} \\
\hline Opción & Frecuencia & Porcentaje \\
\hline En cierta medida & 18 & 15,1 \\
\hline Bastante & 45 & 37,8 \\
\hline Mucho & 56 & 47,1 \\
\hline Total & 119 & 100,0 \\
\hline
\end{tabular}

Fuente: elaboración propia.

Tabla 22. Tabla de frecuencia ítem 15

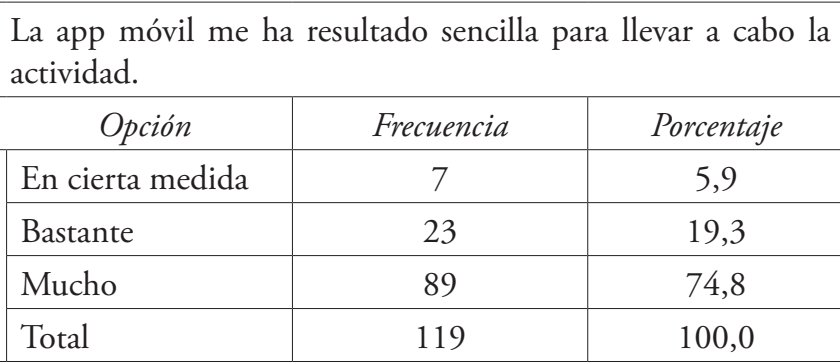

Fuente: elaboración propia.

\section{Dimensión 5: Aporte para futura labor profesional}

Por último, a modo de resultado final de los datos que se extraen de la tabla 23, se puede inferir que la actividad le ha aportado al alumnado una experiencia moderada, ya que el rango de respuestas es grande, y la moda no es el número más alto. No 
obstante, analizando la tabla 24, se puede ver que los mayores porcentajes son los de las opciones Bastante y $M u c h o$, por lo que pensamos que, en líneas generales, los alumnos están satisfechos con la actividad y, por consiguiente, pensarían que la podrían aplicar en un futuro contexto profesional.

Tabla 23. Datos estadísticos descriptivos de la dimensión: Aporte para futura labor profesional

\begin{tabular}{|c|c|c|c|c|c|c|c|}
\hline \multicolumn{1}{|c|}{ Ítems } & \multicolumn{2}{|c|}{$N$} & \multirow{2}{*}{ Media } & Moda & $\begin{array}{c}\text { Desviación } \\
\text { estándar }\end{array}$ & Mínimo & Máximo \\
\cline { 2 - 6 } & Válido & Perdidos & & & & & \\
\hline $\begin{array}{l}\text { A modo de } \\
\text { resultado } \\
\text { general, la activ- } \\
\text { idad me ha } \\
\text { aportado una } \\
\text { experiencia que } \\
\text { podría aplicar } \\
\text { en mi futuro } \\
\text { contexto profe- } \\
\text { sional. }\end{array}$ & 119 & 0 & 4,29 & 4 &, 668 & 2 & 5 \\
\hline
\end{tabular}

Fuente: elaboración propia.

Tabla 24. Tabla de frecuencia ítem 16

A modo de resultado general, la actividad me ha aportado una experiencia que podría aplicar en mi futuro contexto profesional.

\begin{tabular}{|l|c|c|}
\hline \multicolumn{1}{|c|}{ Opción } & Frecuencia & Porcentaje \\
\hline Poco & 1 &, 8 \\
\hline En cierta medida & 11 & 9,2 \\
\hline Bastante & 59 & 49,6 \\
\hline Mucho & 48 & 40,3 \\
\hline Total & 119 & 100,0 \\
\hline
\end{tabular}

Fuente: elaboración propia.

Por último, para acabar la exposición de los datos descriptivos y de frecuencias, nos parece importante comentar la composición de las menciones en relación con el género. Todos son grupos bastante similares en cuanto número de componentes, predominando el género femenino en todos ellos de manera importante:
Tabla 25. Tabla cruzada género y mención de pertenencia

\begin{tabular}{|c|c|c|c|c|c|}
\hline & Francés & Inglés & Intercultural & Total \\
\hline \multirow{2}{*}{ 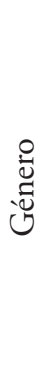 } & 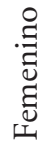 & 29 & 31 & 38 & 98 \\
\hline & 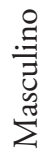 & 4 & 7 & 10 & 21 \\
\hline \multicolumn{2}{|c|}{ Total } & 33 & 38 & 48 & 119 \\
\hline
\end{tabular}

Fuente: elaboración propia.

Por otro lado, se ha realizado el agrupamiento de variables pertenecientes a las dimensiones estudiadas, asignando cinco valores cualitativos alfanuméricos, según el valor numérico que pudiese producir cada variable. Estos valores han sido cinco: nada valorado, poco valorado, algo valorado, bastante valorado, muy valorado. Los rangos que no aparecen son porque no tienen ningún valor asignado. En las tablas 26, 27, 28,29 y 30 , se pueden ver de forma global las valoraciones cualitativas de las dimensiones estudiadas, ofreciendo una visión amplia de la percepción general que se le ha atribuido a la práctica realizada.

Tabla 26. Tabla cruzada mención de pertenencia e intervalos de valor en Dimensión 1

\begin{tabular}{||c|c|c|c|c|}
\hline \multirow{2}{*}{} & \multicolumn{3}{|c|}{$\begin{array}{c}\text { Valoración Dimensión 1 } \\
\text { (Interacción del alumno con la } \\
\text { obra) }\end{array}$} & \\
\cline { 2 - 5 } & $\begin{array}{c}\text { Algo } \\
\text { valorado }\end{array}$ & $\begin{array}{c}\text { Bastante } \\
\text { valorado }\end{array}$ & $\begin{array}{c}\text { Muy } \\
\text { valorado }\end{array}$ & Total \\
\hline Francés & 1 & 15 & 17 & 33 \\
\hline Inglés & 4 & 15 & 19 & 38 \\
\hline Intercultural & 3 & 16 & 29 & 48 \\
\hline Total & 8 & 46 & 65 & 119 \\
\hline
\end{tabular}

Fuente: elaboración propia. 
Tabla 27. Tabla cruzada mención de pertenencia e intervalos de valor en Dimensión 2

\begin{tabular}{|c|c|c|c|c|}
\hline & \multicolumn{3}{|c|}{$\begin{array}{c}\text { Valoración Dimensión } 2 \\
\text { (Interacción del alumno con el } \\
\text { espacio) }\end{array}$} & \multirow[b]{2}{*}{ Total } \\
\hline & Valorado & $\begin{array}{l}\text { Bastante } \\
\text { valorado }\end{array}$ & $\begin{array}{c}\text { Muy } \\
\text { valorado }\end{array}$ & \\
\hline Francés & 2 & 17 & 14 & 33 \\
\hline Inglés & 5 & 7 & 26 & 38 \\
\hline Intercultural & 0 & 24 & 24 & 48 \\
\hline Total & 7 & 48 & 64 & 119 \\
\hline
\end{tabular}

Fuente: elaboración propia.

Tabla 28. Tabla cruzada mención de pertenencia e intervalos de valor en Dimensión 3

\begin{tabular}{||c|c|c|c|c|}
\hline \multirow{2}{*}{} & \multicolumn{3}{|c|}{$\begin{array}{c}\text { Valoración Dimensión 3 } \\
\text { (Interacción con las personas) }\end{array}$} & \multirow{2}{*}{ Total } \\
\cline { 2 - 4 } & $\begin{array}{c}\text { Algo } \\
\text { valorado }\end{array}$ & $\begin{array}{c}\text { Bastante } \\
\text { valorado }\end{array}$ & $\begin{array}{c}\text { Muy } \\
\text { valorado }\end{array}$ & \\
\hline Francés & 1 & 8 & 24 & 33 \\
\hline Inglés & 0 & 17 & 21 & 38 \\
\hline Intercultural & 0 & 15 & 33 & 48 \\
\hline Total & 1 & 40 & 78 & 119 \\
\hline
\end{tabular}

Fuente: elaboración propia.

Tabla 29. Tabla cruzada mención de pertenencia e intervalos de valor en Dimensión 4

\begin{tabular}{||c|c|c|c|c|}
\hline \multirow{2}{*}{} & \multicolumn{3}{|c|}{$\begin{array}{c}\text { Valoración Dimensión 4 } \\
\text { (Interacción con el dispositivo } \\
\text { móvil) }\end{array}$} \\
\cline { 2 - 5 } & $\begin{array}{c}\text { Algo } \\
\text { valorado }\end{array}$ & $\begin{array}{c}\text { Bastante } \\
\text { valorado }\end{array}$ & $\begin{array}{c}\text { Muy } \\
\text { valorado }\end{array}$ & \multirow{2}{*}{ Total } \\
\hline Francés & 0 & 8 & 25 & 33 \\
\hline Inglés & 6 & 12 & 20 & 38 \\
\hline Intercultural & 0 & 11 & 37 & 48 \\
\hline Total & 6 & 31 & 82 & 119 \\
\hline
\end{tabular}

Fuente: elaboración propia.
Tabla 30. Tabla cruzada mención de pertenencia e intervalos de valor en Dimensión 5

\begin{tabular}{|l|c|c|c|c|c|}
\hline \multirow{2}{*}{} & \multicolumn{4}{|c|}{ Valoración Dimensión 5 } & \multirow{2}{*}{ Total } \\
\cline { 2 - 5 } & $\begin{array}{c}\text { (Aporte para futura labor profesional) } \\
\text { valorado }\end{array}$ & $\begin{array}{c}\text { Algo } \\
\text { valorado }\end{array}$ & $\begin{array}{c}\text { Bastante } \\
\text { valorado }\end{array}$ & $\begin{array}{c}\text { Muy } \\
\text { valorado }\end{array}$ & \\
\hline Francés & 0 & 2 & 15 & 16 & 33 \\
\hline Inglés & 1 & 7 & 18 & 12 & 38 \\
\hline $\begin{array}{l}\text { Inter- } \\
\text { cultural }\end{array}$ & 0 & 2 & 26 & 20 & 48 \\
\hline Total & 1 & 11 & 59 & 48 & 119 \\
\hline
\end{tabular}

Fuente: elaboración propia.

De forma general, los datos analizados poseen un carácter bastante positivo, con gran cantidad de valoraciones distribuidas entre las dos opciones de valoración más altas de la escala Likert. Ello supone que el planteamiento de la actividad educativa, en un contexto tan complejo como el artístico, en el que lo perceptivo trabaja de forma inseparable a los espacios expositivos y a las obras, además, utilizando los dispositivos móviles como mediadores para llevar a cabo los diálogos, ha producido una experiencia educativa valiosa.

\section{Conclusiones y discusión}

Tras la valoración de los resultados del cuestionario realizado por el alumnado, podemos llegar a las siguientes conclusiones:

El objetivo planteado se pudo realizar de forma completa. Las cinco dimensiones analizadas ofrecen unos resultados altamente satisfactorios: la Dimensión 1 (interacción del alumno con la obra) está entre bastante y muy valorada por el 93,3\% de los alumnos; la Dimensión 2 (interacción del alumno con el espacio) por un 94,1 \%; la Dimensión 3 (interacción con las personas) por un 99,1 \%; la Dimensión 4 (interacción con el dispositivo móvil) por un 94,9 \%; y la Dimensión 5 (aporta para futura labor 
profesional) por un $89,9 \%$, porcentajes corroborados por unos índices de desviación estándar por debajo de 1 , lo que se interpreta como bastante unanimidad de criterio. Estos datos certifican que la metodología empleada hacia estudiantes universitarios sobre unos espacios expositivos tan particulares, ha sido acertada. Así, podríamos considerar factible aplicar técnicas para el aprendizaje situado en contextos artísticos expositivos desde premisas de carácter cognitivo. Además, se observa que el alumnado se implicó en la actividad de forma generalizada, debido, principalmente, a lo atractivo del conjunto de interacciones que se propusieron, y que después se evaluaron. Se ha podido comprobar que, para el alumnado, resulta motivadora y satisfactoria la opción de trabajar con una herramienta con la que está tan familiarizado, y es utilizada con tanta asiduidad.

Si revisamos otros trabajos previos, como el de Melgar y Donolo (2011) en el que se muestra una interacción del alumnado con el museo, podemos alcanzar unas conclusiones similares, incluidas las que expresan en relación con la pertinencia de que los alumnos fuesen más a menudo a los mismos escenarios, pudiendo realizar así actividades previas y posteriores a las visitas. En este sentido, reconocemos que hay una falta de asiduidad de visita a museos de nuestros alumnos, especialmente desde una perspectiva curricular. Coincidimos con estos autores en el uso que hacen del museo como espacio para observar a sus alumnos, para "conocer qué les interesa, qué los sorprende, cuáles son sus capacidades más destacadas, cómo se relacionan los alumnos en un nuevo espacio y con sus compañeros, entre otros aspectos" (Melgar y Donolo, 2011, p. 324).

Respecto a la utilización de una app móvil en el museo como herramienta educativa, López y Santacana (2015) proponen el uso de los recursos como forma de intermediar e interpretar a través de apps sin obstaculizar el pensamiento crítico, tal y como trabajamos en este artículo. Es decir, "es necesario impulsar a través de las aplicaciones para portátiles o móviles para su uso didáctico pero trabajado el docente como mediador" (López y Santacana, 2015, p. 9).
Un estudio muy similar al presente es el de Hou, Wu, Lin, Sung, Lin y Chang (2014), que lo efectúan en museos de semejantes características, donde interactúan con 54 alumnos. En dicho trabajo se realizó un análisis de rendimiento, un análisis de comportamiento de la participación de los alumnos en el sitio web y un análisis secuencial de los comportamientos grabados en video de los participantes visitantes. Los resultados apoyan el trabajo que hemos realizado, distribuido sobre las dimensiones: interacción del alumno con la obra, interacción del alumno con el espacio museístico, interacción del propio alumno entre sí, interacción con el dispositivo y aporte de la actividad para su futura labor profesional, por lo que se podría decir que los resultados que hemos obtenido se ven respaldados por el trabajo de estos investigadores mencionados.

Un trabajo muy similar es el de Mancinas (2018), en el que muestra los resultados de una investigación en función de los teléfonos y las modalidades de cognición involucradas; asimismo, el estudio de Dickstein y Tobin (2018), donde además de utilizar recorridos conversacionales en museos, se proponen actividades de comprensión basadas en la atención plena y en ejercicios de creación artística, desarrollando el pensamiento crítico y visual. Estas dos investigaciones, a la que podríamos sumar la de Cahill et al. (2011), corresponden de forma directa con los resultados de la investigación realizada, apoyando de una manera clara, la metodología empleada.

Los recursos espaciales utilizados, un dispositivo móvil y una app, quizás sean herramientas que poseen ciertas connotaciones negativas para que un maestro pueda utilizar con nińos de los primeros cursos, pero para niños con edades algo más avanzadas, tal y como recomienda la UNESCO (2019), es un planteamiento que perfectamente se pudiera integrar en la práctica educativa habitual del profesor. Es precisamente en este punto donde se encuentran las vías de continuación, por un lado, a través de la creación, adaptación y utilización de nuevos recursos TIC, y por el otro, la aplicación de nuevas variantes del aprendizaje en contextos artísticos. 


\section{Referencias}

Aguayo, C., Eames, C. y Cochranea, T. (2020). A Framework for Mixed Reality Free-Choice, Self-Determined. Research in Learning Technology, 28. Doi: http://dx.doi.org/10.25304/rlt. v28.2347

Alcaide, J. (2019). Pepe Espaliú - Juan Muñoz. Correspondencias. Recuperadode: http://www.salaveronicas. es/servlet/s.Sl?sit=c, 893, m,3775,i,1\&r=Portal-1043-DETALLE_EXP

Bernabéu, E. (2017). La atención y la memoria como claves del proceso de aprendizaje. Aplicaciones para el entorno escolar. ReiDoCrea, 6(2), 16-23.

Cahill, C., Kuhn, A., Schmoll, S., Lo, W., McNally, B. y Quintana, C. (2011). Mobile Learning in Museums: How Mobile Supports for Learning Influence Student. Actas de la Décima Conferencia Internacional sobre Diseño de Interacción y Niños. 21-28. Recuperado de https:// doi.org/10.1145/1999030.1999033

Carrión, J. (2019, 3 de septiembre). Modigliani viaja a Murcia con los artistas más ocultos de la Escuela de París. La Verdad. Recuperado de https://bit. ly/30YW9Jf

Dickstein, L. y Tobin, A. (2018). ArtInSight: A Contemplative Approach to Museum Gallery Teaching and Learning. Journal of Museum Education, 43(4), 334-341. Doi: $10.1080 / 10598650.2018 .1512820$

Engenström, Y. (1987). Learning by expanding: An activity-theoretical approach to development research. Helsinki: Orienta-Konsultit. Recuperado de: http://lchc.ucsd.edu/mca/Paper/ Engestrom/Learning-by-Expanding.pdf

Gabriel Pedro, L. F. M., Oliveira Barbosa, C. M. M., y Neves Santos, C. M. (2018). A critical review of mobile learning integration in formal educational contexts. Int J EducTechnol High Educ 15, 10. Doi: 10.1186/s41239-018-0091-4

Hou, H. T., Wu, S. Y., Lin, P. C., Sung, Y. T., Lin, J. W. y Chang, K. E. (2014). A Blended Mobile
Learning Environment for Museum Learning. Educational Technology \& Society, 17(2), 207-218.

Huerta, R. (2019). Arte para primaria. Valencia: UOC.

Kali, Y., Sagy, O., Kuflik, T., Mogilevsky, O. y Maayan-Fanar, E. (2015). Harnessing Technology for Promoting Undergraduate Art Education: A Novel Model that Streamlines Learning between Classroom, Museum, and Home. Learning Technologies, IEEE Transactions, 8(1), 5-17. Doi: 10.1109/TLT.2014.2365810

Kearney M., Burden K. y Schuck S. (2019). Disrupting Education Using Smart Mobile Pedagogies. In: L. Daniela (ed.). Didactics of Smart Pedagogy. Cham: Springer. Doi: 10.1007/9783-030-01551-0_7

Keskin, N. y Metcalf, D. (2011). The current perspectivas, theories and practices of mobile learning. Turkish Online Journal of Educational Technology, 10(2). Recuperado de http://eric. ed.gov/?id=EJ932239

Koole, M. (2009). A Model for Framing Mobile Learning. En M. Ally (Ed.), Mobile Learning: Transforming the Delivery of Education and Training (pp. 25-47). Edmonton: Athabasca University.

Laurillard, D. (2007). Pedagogical forms of mobile learning: framing research questions. En N. Pachler (ed.) Mobile learning: towards a research agenda. London: WLE Centre, Institute of Education.

López, V. y Santacana, J. (2015). Cultura digital, museos y educación. Her\&Mus. Heritage \& Museography, 13, 8-15.

Mancinas, A. (2018). Modalidades de cognición en un curso universitario basado en el aprendizaje móvil. Apertura, 10(1), 1-20. Doi: 10.32870/ Ap.v10n1.1159

Melgar, M. y Donolo, D. (2011). Salir del aula... Aprender de otros contextos: Patrimonio natural, museos e Internet. Revista Eureka sobre Enseñanza y Divulgación de las Ciencias, 8(3), 323-333. Doi: 10498/14396 
Ministerio de Cultura y Deporte (2019). Encuesta de hábitos y prácticas culturales 2018-2019. Recuperado de https://www.culturaydeporte.gob. es/servicios-al-ciudadano/estadisticas/cultura/ mc/ehc/2018-2019/presentacion.html

Naismith, L., Lonsdale, P., Vavoula, G. y Sharples, M. (2004). Literature Review in Mobile Technologies and Learning. Recuperado de https:// www.nfer.ac.uk/media/1779/futl15.pdf

Park, Y. (2011). A Pedagogical Framework for Mobile Learning: Categorizing Educational Applications of Mobile Technologies into Four Types. International Review of Research in Open and Distance Learning, 12. Doi: https:/doi. org/10.19173/irrodl.v12i2.791

Patten, B., Arnedillo, I. y Tangney, B. (2006). Designing collaborative, constructionist and contextual applications for handled devices. Computers \& Education, 46(3), 294-308. Doi: 10.1016/j.compedu.2005.11.011

Poikela, S., Vuoskoski, P. y Kärnä, M. (2009). Developing Creative Learning Environments in Problem-based Learning. En O. Tan (ed.), Problem-Based Learning and Creativity (pp. 67-87). Singapore: Cengage Learning.

Rainoldi, M., Neuhofer B., y Jooss, M. (2018). Mobile eyetracking of museum learning experiences. En B. Stangl, y J. Pesonen (eds.), Information and Communication Technologies in Tourism 2018, (pp. 473-485). Cham: Springer.

Rickenmann, R. (2007). El rol de los artefactos culturales en la estructuración y gestión de secuencias de enseñanza-aprendizaje. Conferencia Seminario Doctoral. Universidad Pedagógica Nacional, Bogotá. Recuperado de http://www.unige.ch/ fapse/clidi/textos/artefactos-culturales-RR.pdf

Rosas Mantecón, R. (2017). Públicos: historia y contemporaneidad. En L. Pérez Castellanos (ed.), Estudios sobre públicos y museos, 3. México: ENCRyM

Sabariego, M. y Bisquerra, R. (2009). El proceso de investigación (parte 1). En R. Bisquerra (ed.). Metodología de la investigación educativa (pp. 89-125). Madrid: La Muralla.
Sandoval, W. (2014). Conjecture Mapping: An Approach to Systematic Educational Design Research. Journalof the Learning Sciences, 23(1), 18-36. Doi: 10.1080/10508406.2013.778204

Sharples, M., Taylor, J. y Vavoula, G. (2007). A Theory of Learning for the Mobile Age. En R. Andrews y C. Haythornthwaite (eds.). The Sage Handbook of Elearning Research, (pp. 221-247). London: Sage.

Socrative (2020). Socrative. Recuperado de https:// socrative.com/

UNESCO (2019). Mobile learning. Recuperado de https://en.unesco.org/themes/ict-education/ mobile-learning

Vieytes, R. (2004). Metodología de la investigación en organizaciones, mercado y sociedad. Epistemología y Técnicas. Buenos Aires: Editorial de las Ciencias.

Vygotsky, L. (1978). Mind and society: The development of higher mental processes. En M. Cole, V. John-Steiner, S. Scribner y E. Souberman (eds.). Cambridge, MA: Harvard University Press.

Wang, M. y Shen, R. (2012). Message design for mobile learning: learning theories, human cognition and design principles. British Journal of Educational Technology, 43, 561-575. Doi: 10.1111/j.1467-8535.2011.01214.x

Welford A. T. (1952). The 'psychological refractory period' and the timing of high-speed performance: a review and a theory. British Journal of Psychology, 43, 2-19.

Zapata-Ros, M. (2015). Teorías y modelos sobre el aprendizaje en entornos conectados y ubicuos. Bases para un nuevo modelo teórico a partir de una visión crítica del "conectivismo". Education In The Knowledge Society (EKS), 16(1), 69-102. Doi: http://dx.doi.org/10.14201/ eks201516169102 